\title{
Pancreatic Necrosis, CTCAE
}

National Cancer Institute

\section{Source}

National Cancer Institute. Pancreatic Necrosis, CTCAE. NCI Thesaurus. Code C143732.

A disorder characterized by a necrotic process occurring in the pancreas. 